\title{
Critical behavior of self-assembled rigid rods on triangular and honeycomb lattices
}

\author{
L. G. López, D. H. Linares, and A. J. Ramirez-Pastor* \\ Departamento de Física, Instituto de Física Aplicada, \\ Universidad Nacional de San Luis, CONICET, 5700 San Luis, Argentina
}

(Dated: August 3, 2018)

\begin{abstract}
Using Monte Carlo simulations and finite-size scaling analysis, the critical behavior of selfassembled rigid rods on triangular and honeycomb lattices at intermediate density has been studied. The system is composed of monomers with two attractive (sticky) poles that, by decreasing temperature or increasing density, polymerize reversibly into chains with three allowed directions and, at the same time, undergo a continuous isotropic-nematic IN transition. The determination of the critical exponents, along with the behavior of Binder cumulants, indicate that the IN transition belongs to the $q=1$ Potts universality class.

PACS numbers: $05.50 .+\mathrm{q}, 64.70 . \mathrm{Md}, 75.40 . \mathrm{Mg}$
\end{abstract}

\section{INTRODUCTION}

Self-assembly has been considered for over 50 years to be central to understanding structure formation in living systems [1]. As a consequence, an increasing interest has been devoted to enhance our understanding of the theoretical basis of the fundamental mechanisms governing self-assembly and the observables required to characterize the interactions driving thermodynamic self-assembly transitions [2 9]. Despite these studies, the knowledge of how this process works is still incomplete and many of the basic principles characterizing this type of organization are unclear.

It is obvious that a complete analysis of the selfassembly phenomenon is a quite difficult subject because of the complexity of the involved microscopic mechanisms. For this reason, the understanding of simple models with increasing complexity might be a help and a guide to establish a general framework for the study of this kind of systems, and to stimulate the development of more sophisticated models which can be able to reproduce concrete experimental situations.

In this line of work, two previous articles [8, 9], referred to as papers I and II, respectively, were devoted to the study of a system of self-assembled rigid rods adsorbed on a two-dimensional lattice. In paper I, Tavares et al. studied a system composed of monomers with two attractive (sticky) poles that polymerize reversibly into polydisperse chains and, at the same time, undergo an isotropic-nematic (IN) continuous phase transition. So, the interplay between the self-assembly process and the nematic ordering is a distinctive characteristic of these systems. Using an approach in the spirit of the Zwanzig model [10], the authors found that nematic ordering enhances bonding. In addition, the average rod length was described quantitatively in both phases, while the location of the ordering transition, which was found to be continuous, was predicted semiquantitatively by the the-

*Electronic address: antorami@unsl.edu.ar ory. With respect to the characteristics of the phase transition, Tavares et al. assumed as working hypothesis that the nature of the IN transition remains unchanged with respect to the case of monodisperse rigid rods on square lattices, where the transition is in the 2D Ising universality class 11 13.

Paper II was a step further, analyzing the universality class of the IN phase transition at intermediate density. For this purpose, an extensive work of Monte Carlo (MC) simulations and finite-size scaling (FSS) analysis was carried out. The obtained results showed that the self-assembly process affects the nature of the transition. Thus, the accurate determination of the critical exponents indicated that, for a square lattice, the universality class of the IN transition changes from 2D Ising-type for monodisperse rods without self-assembly [12] to $q=1$ Potts-type for self-assembled rods.

In this context, the objectives of the present paper are (1) to extend the previous work to triangular and honeycomb lattices using the same techniques developed in paper II; and (2) to study the effect of the lattice structure on the critical behavior of self-assembled rigid rods. For this purpose, MC simulations [14] supplemented by analysis using FSS theory [15] have been carried out to study the critical behavior in a system of self-assembled rigid rods deposited on triangular and honeycomb lattices. As in paper II, the calculations were developed at constant temperature and different densities, being $\left(\theta / \theta_{c}-1\right)$ the normalized scaling variable, where $\theta$ and $\theta_{c}$ represent density and critical density, respectively. The accurate determination of the critical exponents, along with the behavior of Binder cumulants, confirmed that the IN transition of self-assembled rigid rods on triangular and honeycomb lattices at intermediate density belongs to the $q=1$ Potts universality class. The outline of the paper is as follows. In Sec. II we describe the lattice-gas model and the simulation scheme. In Sec. III we present the MC results and the general conclusions. 


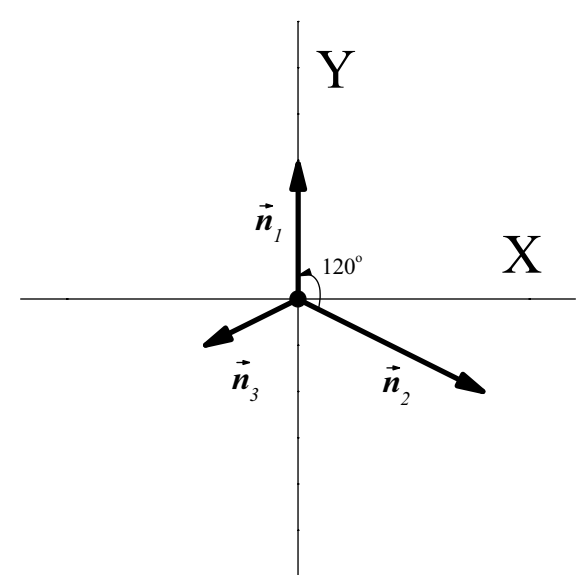

(a)

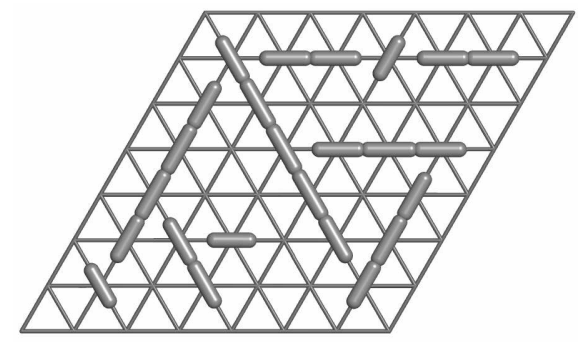

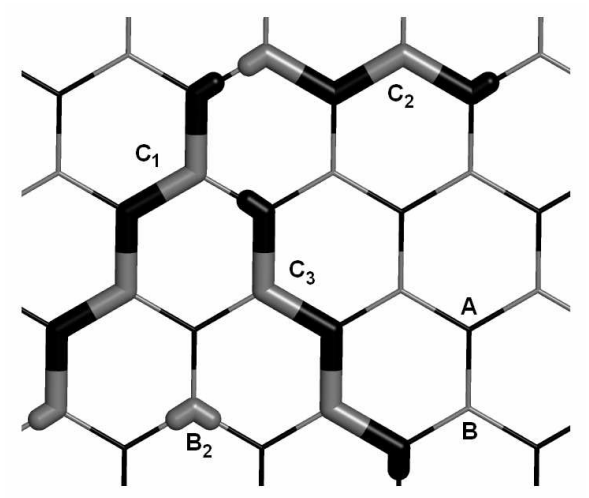

(c)

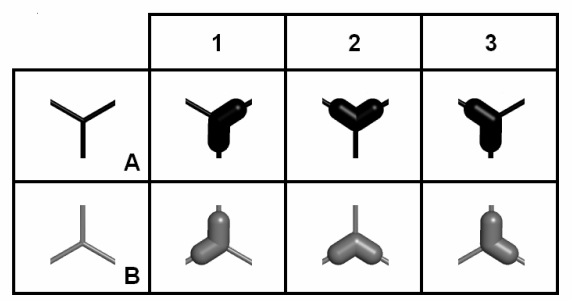

(b)

(d)

FIG. 1: (a) Schematic representation of the set of vectors $\left\{\vec{n}_{1}, \vec{n}_{2}, \vec{n}_{3}\right\}$ for triangular and honeycomb lattices. (b) Self-assembled rigid rods on triangular lattices. (c) Self-assembled rigid rods on honeycomb lattices. See discussion in the text. (d) Available configurations for a monomer on a honeycomb lattice.

\section{LATTICE-GAS MODEL AND MONTE CARLO SIMULATION SCHEME}

As in papers I and II, we consider a system of selfassembled rods with a discrete number of orientations in two dimensions. In this case, the surface is represented as an array of $M=L \times L$ adsorption sites in a triangular or in a honeycomb lattice arrangement and periodic boundary conditions. $N$ particles are adsorbed on the substrate with three possible orientations along the principal axis of the lattice. These particles interact with nearest-neighbors (NN) through anisotropic attractive interactions. Thus, a cluster or uninterrupted sequence of bonded particles is a self-assembled linear rod.

Under these considerations, the adsorbed phase is characterized by the Hamiltonian

$$
H=-w \sum_{\langle i, j\rangle}\left|\vec{r}_{i j} \cdot \vec{\sigma}_{i}\right|\left|\vec{r}_{j i} \cdot \vec{\sigma}_{j}\right| \operatorname{div} 1
$$

where $\langle i, j\rangle$ indicates a sum over NN sites; div means integer division; $w$ represents the $\mathrm{NN}$ lateral interaction between two neighboring $i$ and $j$, which are aligned with each other and with the intermolecular vector $\vec{r}_{i j}$; and $\vec{\sigma}_{i}$ is the occupation vector with $\vec{\sigma}_{i}=0$ if the site $i$ is empty, $\vec{\sigma}_{i}=\hat{x}_{1}$ if the site $i$ is occupied by a particle with orientation along the $x_{1}$-axis, $\vec{\sigma}_{i}=\hat{x_{2}}$ if the site $i$ is occupied by a particle with orientation along the $x_{2}$-axis, and $\vec{\sigma}_{i}=\hat{x_{3}}$ if the site $i$ is occupied by a particle with orientation along the $x_{3}$-axis.

At fixed temperature, the average rod length increases as the density increases and the polydisperse rods will undergo an nematic ordering transition [8, 9]. In order to follow the formation of the nematic phase, we use the order parameter defined in previous work [8, 9], which in this case can be written as

$$
\delta=\frac{\left|\vec{n}_{1}+\vec{n}_{2}+\vec{n}_{3}\right|}{\left|\vec{n}_{1}\right|+\left|\vec{n}_{2}\right|+\left|\vec{n}_{3}\right|}
$$

where each vector $\vec{n}_{m}$ is associated to one of the 3 possible orientations (or directions) for a chain on the lattice. In addition, (1) the $\vec{n}_{i}$ 's lie in the same plane (or are coplanar) and point radially outward from a given point $P$ which is defined as coordinate origin; (2) the angle between two consecutive vectors, $\vec{n}_{i}$ and $\vec{n}_{i+1}$, is equal to $2 \pi / 3$; and (3) the magnitude of $\vec{n}_{i}$ is equal to the number of $k$-mers aligned along the $i$-direction [see Fig. 1(a)]. Note that the $\vec{n}_{i}$ 's have the same directions as the $q$ vectors in Ref. [16]. These directions are not coincident with the allowed directions for the chains on the real lattice.

The concept of linear rod is trivial for triangular lattices [see Fig. 1(b)]. However, in a honeycomb lattice, 
the geometry does not allow for the existence of a linear array of monomers. In this case, we call "linear rod" to a chain of adjacent monomers that can be assembled in only three types of sequences, defining three directions in similar way to the triangular lattice [see Fig. 1(c)]. Two "types" of sites can be recognized in the honeycomb lattice: $A$ (grey) and $B$ (black). Monomers (colored according to the adsorption site) can be placed on these sites in three different orientations: $A_{1}, A_{2}$, and $A_{3}$ (for the $A$-sites); and $B_{1}, B_{2}$, and $B_{3}$ (for the $B$-sites) as shown in Fig. 1(d). So, the self-assembled chains are formed by alternating $A$ and $B$ monomers. In the particular case shown in Fig. 1(c), $C_{1}, C_{2}$, and $C_{3}$ are built by the following sequences: $B_{1} A_{1} B_{1} A_{1} B_{1} A_{1}\left(C_{1}\right), B_{2} A_{2} B_{2} A_{2}$ $\left(C_{2}\right)$, and $A_{3} B_{3} A_{3} B_{3} A_{3}\left(C_{3}\right)$. The figure also shows an unassociated $B$-monomer $\left(B_{2}\right)$.

The problem has been studied by canonical Monte Carlo simulations using an vacancy-particle-exchange Kawasaki dynamics [17] and Metropolis acceptance probability [18]. Typically, the equilibrium state can be well reproduced after discarding the first $10^{7}$ Monte Carlo steps MCS. Then, the next $10^{9}$ MCS are used to compute averages. All calculations were carried out using the parallel cluster BACO located at Instituto de Física Aplicada, Universidad Nacional de San Luis-CONICET, San Luis, Argentina. This facility consists of 60 personal computers each with a $3.0 \mathrm{GHz}$ Pentium- 4 processor and 90 personal computers each with a $2.4 \mathrm{GHz}$ Core 2 Quad processor.

In our Monte Carlo simulations, we set the temperature $T$, varied the density $\theta=N / M$ and monitored the order parameter $\delta$, which can be calculated as simple average. The quantities related with the order parameter, such as the susceptibility $\chi$, and the reduced fourth-order cumulant $U_{L}$ introduced by Binder [14] were calculated as:

$$
\chi=\frac{L^{2}}{k_{B} T}\left[\left\langle\delta^{2}\right\rangle-\langle\delta\rangle^{2}\right]
$$

and

$$
U_{L}=1-\frac{\left\langle\delta^{4}\right\rangle}{3\left\langle\delta^{2}\right\rangle^{2}}
$$

where $\langle\cdots\rangle$ means the average over the MC simulation runs.

In addition, in order to discuss the nature of the phase transition, the fourth-order energy cumulant $U_{E}$ was obtained as

$$
U_{E}=1-\frac{\left\langle H^{4}\right\rangle}{3\left\langle H^{2}\right\rangle^{2}}
$$

\section{RESULTS AND CONCLUSIONS}

As discussed in section I, the phase diagram of a system of self-assembled rigid rods on square lattices has
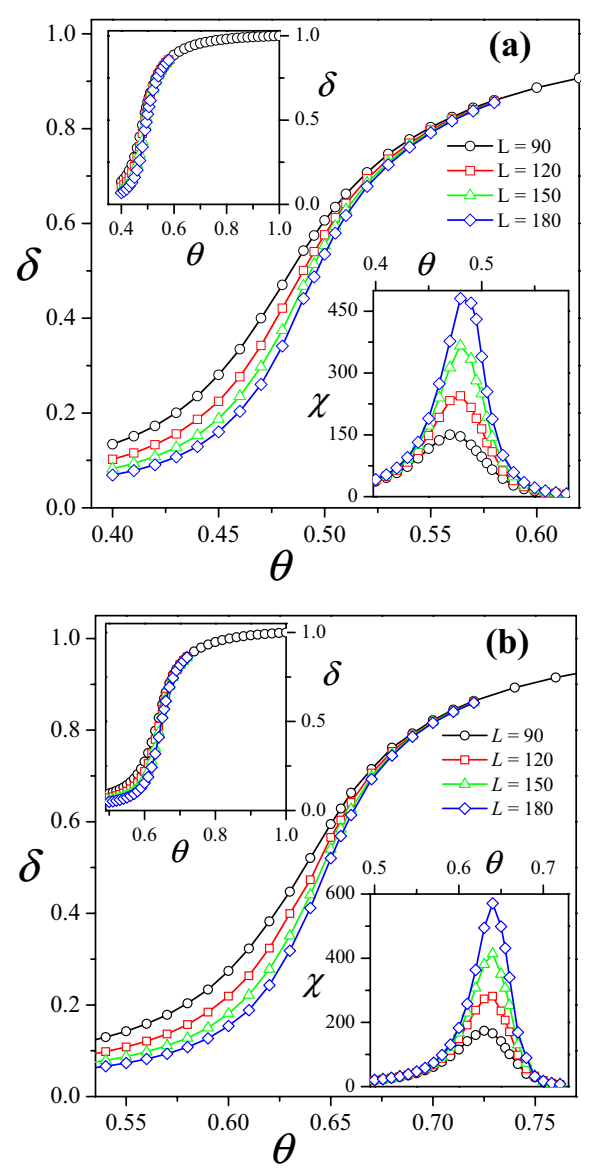

FIG. 2: Size dependence of the order parameter as a function of coverage. Upper-left inset: Order parameter in the regime of high densities. Lower-right inset: Size dependence of the susceptibility as a function of coverage. The error in each measurement is smaller than the size of the symbols. (a) Triangular lattices. (b) Honeycomb lattices.

been recently reported by Tavares et al. [8]. The authors showed that the critical density, at which the IN transition occurs, increases monotonically as $k_{B} T / w$ is increased. Thus, the nematic phase is stable at low temperatures and high densities [see Fig. 1(a) in Ref. [8]]. Later, and based on this finding, the nature of the IN transition at intermediate densities was studied in Ref. [9]. There, the calculations were developed at $w=4 k_{B} T$ and different densities.

In the present study, we set the lateral interaction to $w=4.5 k_{B} T$ [a close value to that used in Ref. [9]], thus allowing a direct comparison with previous results for square lattices. Accordingly, the density was varied around half coverage. For each value of $\theta$, the effect of finite size was investigated by examining lattices with $L$ ranging from 30 to 180 .

We start with the calculation of the order parameter (Fig. 2), and susceptibility (lower-right insets in Fig. 2) plotted versus $\theta$ for several lattice sizes as indicated. Curves in part (a) [(b)] correspond to triangular [hon- 

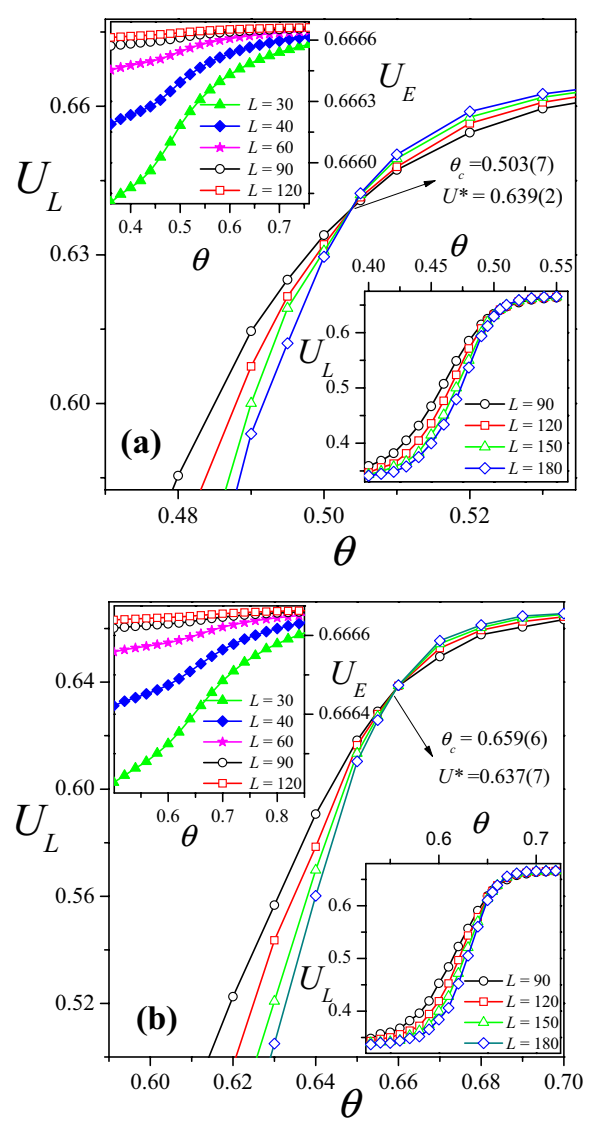

FIG. 3: Curves of $U_{L}(\theta)$ vs $\theta$ for lattices of different sizes. From their intersections one obtained $\theta_{c}$. In the lower-right inset, the data are plotted over a wider range of densities. Upper-left inset: Coverage variation of $U_{E}$ for various lattice sizes. (a) Triangular lattices. (b) Honeycomb lattices.

eycomb] lattices. As it can be observed, $\delta$ appears as a proper order parameter to elucidate the phase transition. When the system is disordered, all orientations are equivalents and $\delta$ is zero. In the critical regime, the particles align along one direction and $\delta$ increases continuously to one, remaining constant up to full coverage (see upper-left insets in Fig. 2, where the order parameter is shown up to $\theta=1$ ). In other words, nematic order survives until $\theta=1$. This finding allows us to discard the existence of a reentrant nematic transition at high densities as speculated in Ref. [8]. With respect to the susceptibility, the curves show a single peak which grows and sharpens as the lattice size is increased.

The critical density has been estimated from the plots of the reduced fourth-order cumulants $U_{L}(\theta)$ plotted vs. $\theta$ for several lattice sizes (see Fig. 3). In the vicinity of the critical point, cumulants show a strong dependence on the system size. However, at the critical point the cumulants adopt a nontrivial value $U^{*}$; irrespective of system sizes in the scaling limit. Thus, plotting $U_{L}(\theta)$ for different linear dimensions yields an intersection point $U^{*}$, which gives an accurate estimation of the critical density in the infinite system. In this case, the values obtained for the critical density were $\theta_{c}=0.503(7)$ for triangular lattices [part (a)] and $\theta_{c}=0.659(6)$ for honeycomb lattices [part (b)].

With respect to the value of the cumulant at the transition density, which allows us to make a "preliminary" identification of the universality class of the transition 14, 19 21], we obtained $U^{*}=0.639(2)$ for triangular lattices [part (a)] and $U^{*}=0.637(7)$ for honeycomb lattices [part (b)]. These values of $U^{*}$ are practically indistinguishable from previous estimates for the twodimensional one-state Potts model (see for instance Ref. [9], where $U^{*}=0.639(3)$ ). This result may be taken as a first indication of universality. In the lower-right inset, the data are plotted over a wider range of densities. As can be seen, the curves exhibit the typical behavior of $U_{L}$ in the presence of a continuous phase transition. Namely, the order-parameter cumulant shows a smooth increase up to its ordered-phase value of $2 / 3$ instead of the characteristic deep (negative) minimum, as in a first-order phase transition 14.

In order to discard the possibility that the phase transition is a first-order one, the energy cumulants [Eq. (9)] have been measured. As is well known, the finite-size analysis of $U_{E}$ is a simple and direct way to determine the order of a phase transition [22 24]. Upper-left inset in Fig. 3 illustrates the energy cumulants plotted versus $\theta$ for different lattice sizes. Data in part (a) correspond to triangular lattices. Data in part (b) correspond to honeycomb lattices. As is observed, $U_{E}$ has the characteristic behavior of a continuous phase transition. Namely, the curves of $U_{E}$ show a dip close to the critical density for all system sizes, but this minimum scales to $2 / 3$ in the thermodynamic limit. This indicates that the latent heat is zero in the thermodynamic limit, which reinforces the arguments given in the paragraphs above.

The results obtained up to here allow us to confirm the existence of a continuous phase transition at intermediate coverage and low temperature. In addition, the evaluation of the fixed point value of the cumulants indicates that, as in the case of square lattices [9], the observed phase transition belongs to the $q=1$ Potts universality class. To corroborate these findings, the critical behavior of the present model has been investigated by means of finite-size scaling (FSS) analysis. The FSS theory implies the following behavior of $\delta, \chi$ and $U_{L}$ at criticality:

$$
\begin{gathered}
\delta=L^{-\beta / \nu} \tilde{\delta}\left(L^{1 / \nu} \epsilon\right), \\
\chi=L^{\gamma / \nu} \tilde{\chi}\left(L^{1 / \nu} \epsilon\right)
\end{gathered}
$$

and

$$
U_{L}=\tilde{U}_{L}\left(L^{1 / \nu} \epsilon\right)
$$

for $L \rightarrow \infty, \epsilon \rightarrow 0$ such that $L^{1 / \nu} \epsilon=$ finite, where $\epsilon \equiv \theta / \theta_{c}-1$. Here $\beta, \gamma$ and $\nu$ are the standard critical exponents of the order parameter $\left(\delta \sim-\epsilon^{\beta}\right.$ for $\epsilon \rightarrow 0^{-}$, 

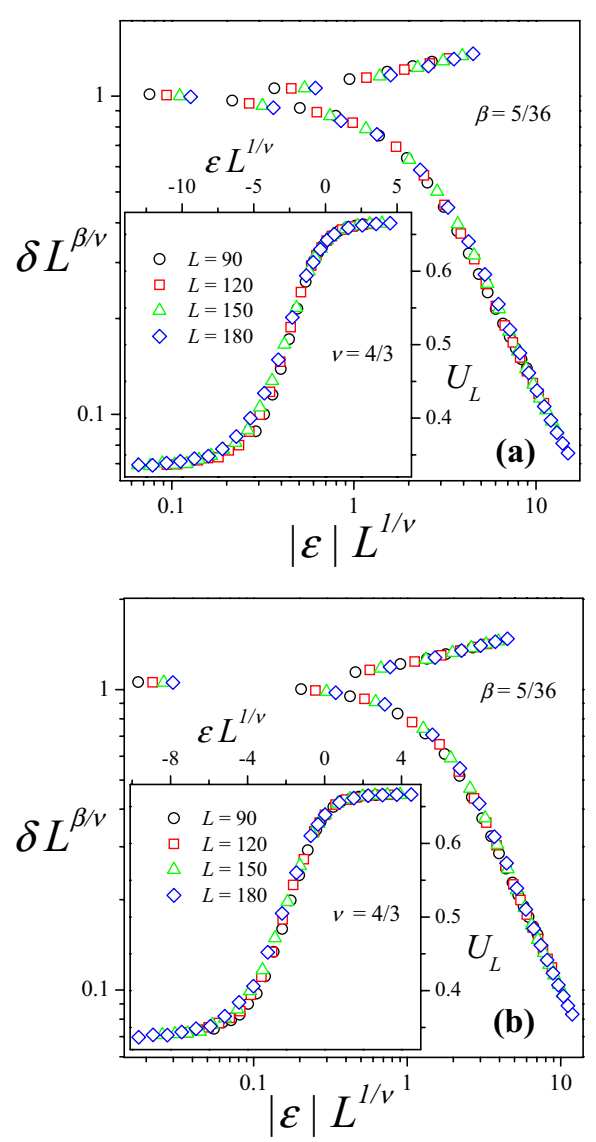

FIG. 4: (a) Data collapsing of the order parameter, $\delta L^{\beta / \nu}$ vs $|\epsilon| L^{1 / \nu}$, and of the cumulant, $U_{L}$ vs $\epsilon L^{1 / \nu}$ (inset) for triangular lattices. The plots were made using $\theta_{c}=0.503$ and the exact percolation exponents $\nu=4 / 3$ and $\beta=5 / 36$. (b) Same as part (a) for honeycomb lattices and $\theta_{c}=0.659$.

$L \rightarrow \infty)$, susceptibility $\left(\chi \sim|\epsilon|^{\gamma}\right.$ for $\left.\epsilon \rightarrow 0, L \rightarrow \infty\right)$ and correlation length $\xi\left(\xi \sim|\epsilon|^{-\nu}\right.$ for $\left.\epsilon \rightarrow 0, L \rightarrow \infty\right)$, respectively. $\tilde{\delta}, \tilde{\chi}$ and $\tilde{U}_{L}$ are scaling functions for the respective quantities.

According to eqs. (6] 8), the scaling behavior can be tested by plotting $\langle\delta\rangle L^{\beta / \nu}$ vs $|\epsilon| L^{1 / \nu}, \chi L^{-\gamma / \nu}$ vs $\epsilon L^{1 / \nu}$, and $U_{L}$ vs $\epsilon L^{1 / \nu}$ and looking for data collapsing. Using the exact values of the critical exponents of the ordinary percolation (one-state Potts model) $\nu=4 / 3, \beta=5 / 36$, and $\gamma=43 / 18$, we obtain an excellent scaling collapse as it is shown in Figs. 4 and 5. Data in part (a) correspond to triangular lattices and $\theta_{c}=0.503$. Data in part (b) correspond to honeycomb lattices and $\theta_{c}=0.659$. The study in Figs. 4 and 5 corroborates that the transition observed corresponds to the universality class of the $q=1$ Potts model.

Finally, it is important to remark that the universality observed for self-assembled rigid rods on triangular and honeycomb lattices coincides with that reported in Ref. [9] for square lattices. In other words, the universality class of the present model does not depend on the lattice geometry. This result (1) reinforces the the arguments
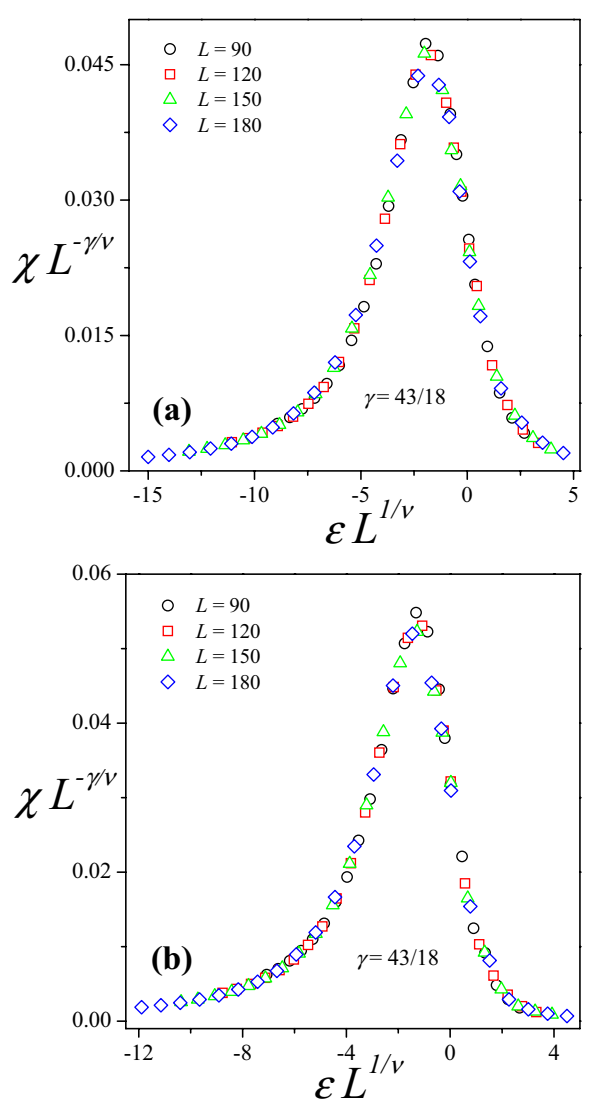

FIG. 5: (a) Data collapsing of the the susceptibility, $\chi L^{-\gamma / \nu}$ vs $\epsilon L^{1 / \nu}$, for the curves in the inset of Fig. 2 (a). The plot was made using $\theta_{c}=0.503$ and the exact percolation exponents $\nu=4 / 3$ and $\gamma=43 / 18$. (b) Same as part (a) for the curves in the inset of Fig. 2 (b) and $\theta_{c}=0.659$.

given in Ref. [9] linking the thermal phase transition (IN phase transition) occurring in the system and the percolation behavior of the clusters of aligned monomers [25]; and (2) reveals a significant difference between the model here discussed and that of monodisperse rigid rods adsorbed on two-dimensional lattices with a discrete number of orientations [11, 12, 26], where the universality class of the IN phase transition occurring at intermediate densities belongs to the 2D Ising universality class for square lattices and to the three-state Potts universality class for triangular and honeycomb lattices.

In summary, we have used Monte Carlo simulations and finite-size scaling theory to study the critical properties of self-assembled rigid rods on triangular and honeycomb lattices at intermediate density. The existence of a IN continuous phase transition was confirmed. In addition, the scaling behavior of the system revealed that, as in the case of square lattices, the phase transition belongs to the $q=1$ Potts universality class. 


\section{Acknowledgments}

This work was supported in part by CONICET (Argentina) under project number PIP 112-200801-01332;
Universidad Nacional de San Luis (Argentina) under project 322000 and the National Agency of Scientific and Technological Promotion (Argentina) under project 33328 PICT 2005.
[1] K. Van Workum and J. F. Douglas, Phys. Rev. E 73, 031502 (2006), and references therein.

[2] P. I. C. Teixeira, J. M. Tavares, and M. M. Telo da Gama, J. Phys.: Condens. Matter 12, R411 (2000).

[3] X. Lü and J. T. Kindt, J. Chem. Phys. 120, 10328 (2004).

[4] Z. Zhang and S. C. Glotzer, Nano Lett. 4, 1407 (2004).

[5] F. Sciortino, E. Bianchi, J. F. Douglas, and P. Tartaglia, J. Chem. Phys. 126, 194903 (2007).

[6] W.-Z. Ouyang and R. Hentschke, Phys. Rev. E 79, 031503 (2009).

[7] J. M. Tavares, P. I. C. Teixeira, and M. M. Telo da Gama, Phys. Rev. E 80, 021506 (2009)

[8] J. M. Tavares, B. Holder, and M. M. Telo da Gama, Phys. Rev. E 79, 021505 (2009).

[9] L. G. López, D. H. Linares, and A. J. Ramirez-Pastor, Phys. Rev. E 80, 040105(R) (2009).

[10] R. Zwanzig, J. Chem. Phys. 39, 1714 (1963).

[11] A. Ghosh and D. Dhar, Eur. Phys. Lett. 78, 20003 (2007).

[12] D. A. Matoz-Fernandez, D. H. Linares, and A. J. Ramirez-Pastor, Europhys. Lett. 82, 50007 (2008).

[13] D. A. Matoz-Fernandez, D. H. Linares, and A. J. Ramirez-Pastor, J. Chem. Phys. 128, 214902 (2008).

[14] A Guide to Monte Carlo Simulations in Statistical Physics, 2nd ed., edited by D. P. Landau and K. Binder (Cambridge University Press, Cambridge, 2005).

[15] Finite Size Scaling and Numerical Simulation of Statistical Systems, edited by Privman V. (World Scientific, Singapore, 1990).
[16] F. Y. Wu, Rev. Mod. Phys. 54, 235 (1982).

[17] K. Kawasaki, in Phase Transitions and Critical Phenomena, edited by C. Domb and M. S. Green (Academic, London, 1972).

[18] N. Metropolis, A.W. Rosenbluth, M.N. Rosenbluth, A.H. Teller and E. Teller, J. Chem. Phys. 21, 1087 (1953).

[19] As recently pointed out by Selke et al. 20, 21], the value of the cumulant intersection may depend on various details of the model, which do not affect the universality class, in particular, the boundary condition, the shape of the lattice, and the anisotropy of the interactions. Consequently, more research is required to determine the universality class of a phase transition.

[20] W. Selke and L. N. Shchur, J. Phys. A: Math. Gen. 38, L739 (2005).

[21] W. Selke, J. Stat. Mech. P04008 (2007).

[22] K. Binder and D. P. Landau, Phys. Rev. B 30, 1477 (1984).

[23] M. S. S. Challa, D. P. Landau and K. Binder, Phys. Rev. B 34, 1841 (1986).

[24] K. Vollmayr, J. D. Reger, M. Scheucher and K. Binder, Z. Phys. B: Condens. Matter 91, 113 (1993).

[25] The appearance of nematic order at critical density is accompanied by the simultaneous presence of a percolating cluster of aligned monomers connecting the extremes of the lattice.

[26] D. A. Matoz-Fernandez, D. H. Linares, and A. J. Ramirez-Pastor, Physica A 387, 6513 (2008). 